\title{
Current status of endoscopic balloon dilation for Crohn's disease
}

\author{
Fumihito Hirai \\ Inflammatory Bowel Disease Center, Fukuoka University Chikushi Hospital, Fukuoka, Japan
}

The therapeutic target in Crohn's disease (CD) has been raised to the achievement of mucosal healing. Although effective treatments that target cytokines and other molecules has been widely used for CD, intestinal strictures are still a major cause of surgery. Endoscopic balloon dilation (EBD) is known to be an effective and safe intervention for intestinal strictures in CD. Since frequent intestinal resection often results in short bowel syndrome and can decrease the quality of life, EBD can help avoid surgery. EBD with a conventional colonoscope for Crohn's strictures of the colon and ileo-colonic anastomosis has established efficacy and safety. In addition, EBD using balloon-assisted enteroscopy has recently been applied for small bowel Crohn's strictures. Although the evidence is not strong, EBD may become an alternative to surgery in small bowel strictures in CD. EBD and other new methods such as self-expanding stent implantation for Crohn's strictures may be useful and safe; however, it is important to address several issues regarding these interventions and to establish a protocol for combined therapies. (Intest Res 2017;15:166-173)

Key Words: Endoscopic balloon dilation; Crohn disease; Colorectal stricture; Ileo-colonic anastomotic stricture; Small bowel stricture

\section{INTRODUCTION}

Crohn's disease (CD) is a chronic inflammatory digestive disease with a long-term course that is usually progressive, disabling, and relapsing, and most patients with CD undergo intestinal surgery during their lifetime. ${ }^{1,2}$ Effective therapy with immunomodulators and anti-tumor necrosis factor $\alpha$ antibody have the potential to change the natural course of CD. Actually, a large cohort study suggested that effective medical treatment has reduced the number of surgeries being performed for $\mathrm{CD}^{3}$ On the other hand, intestinal strictures are still the main reason for surgeries such as intestinal resection and strictureplasty, which are performed to relieve

Received December 29, 2016. Revised December 31, 2016.

Accepted January 2, 2017. Published online March 20, 2017

Correspondence to Fumihito Hirai, Inflammatory Bowel Disease Center,

Fukuoka University Chikushi Hospital, 1-1-1, Zokumyoin, Chikushino,

Fukuoka 818-8502, Japan. Tel: +81-92-921-1011, Fax: 81-92-929-2630,

E-mail:fuhirai@cis.fukuoka-u.ac.jp

Financial support: None. Conflict of interest: None. stricture-related symptoms or ileus. Surgery is, however, not a basic treatment modality, and relapse at the anastomotic site is often a problem in $\mathrm{CD}^{4,5}$ Surgery sometimes lead to stricture recurrence, with the need for repeat surgery, and frequent surgery can lead to short bowel syndrome. ${ }^{6}$ Endoscopic balloon dilation (EBD) is an alternative to surgery for strictures secondary to CD. This endoscopic treatment is mainly indicated for Crohn's strictures of the gastric outlet, duodenum, colon and, ileo-colonic anastomosis. ${ }^{7-24}$ Many reports concluded that EBD is effective and safe, and its use can avoid surgery. Moreover, balloon-assisted enteroscopy (BAE), including double-balloon enteroscopy and singleballoon enteroscopy have been widely used for diagnosis and endoscopic treatment of small bowel diseases. ${ }^{25,26}$ Since BAE enables us to perform EBD for small bowel strictures in patients with $C D$, the indications for EBD in CD have increased. ${ }^{27-34}$ Hence, it is important for gastroenterologists to know the current status and future direction of EBD for Crohn's strictures. This article reviews the literature on EBD performed using the traditional approach to strictures of the

๑ Copyright 2017. Korean Association for the Study of Intestinal Diseases. All rights reserved.

This is an Open Access article distributed under the terms of the Creative Commons Attribution Non-Commercial License (http://creativecommons.org/licenses/by-nc/4.0)

which permits unrestricted non-commercial use, distribution, and reproduction in any medium, provided the original work is properly cited. 
colon and ileo-colonic anastomosis, in addition to that on recent approaches to small bowel strictures using BAE.

\section{EBD FOR COLORECTAL AND ILEO-COLONIC ANASTOMOTIC STRICTURES}

\section{Dilation Technique}

Two different EBD dilation catheters are used for gastrointestinal strictures: an over-the-wire balloon catheter and a through-the-scope balloon catheter. Through-the-scope balloon catheters tend to be selected for almost all EBD procedures because of their simplicity and safety. The length of the balloons for inflation is about $5 \mathrm{~cm}$; therefore, stenoses 5 $\mathrm{cm}$ or longer are considered unsuitable for EBD. Moreover, intestinal strictures with deep ulcers and fistulous complications are contraindication for EBD. The dilation procedure is performed with monitoring of the pressure of the inflated balloon using a dilator under X-ray guidance. The maximum dilation diameter is selected at the discretion of the operating endoscopist. Table 1 shows a summary of published studies (only those that included 15 cases or more) on EBD using conventional colonoscopy in CD patients. ${ }^{8-24}$ In these 17 published studies, five used a maximum dilation diameter of $18 \mathrm{~mm}$, eight used $20 \mathrm{~mm}$, and four used $25 \mathrm{~mm}$. Lower dilation pressure was thought to be a risk factor for earlier redilation in two studies. ${ }^{11,16}$ On the other hand, Gustavsson et $\mathrm{al}^{20}{ }^{20}$ suggested that larger maximum dilation diameter (25 $\mathrm{mm}$ ) in EBD was associated with complications such as perforation and hemorrhage. Therefore, when performing EBD, forcible dilation to achieve a larger dilation diameter or pressure is not recommended, as it could lead to intestinal perforation, and would require surgical intervention. Ideally, we should maximize the less invasive nature of EBD compared to surgery.

\section{Short-Term Outcomes and Safety}

A number of studies have evaluated the technical success and short-term clinical efficacy of EBD for Crohn's strictures. However, there are no standardized definitions of short-term success of EBD. Some authors simply defined successful dilation as technical response, while others defined successful dilation as disappearance of stenotic symptoms or ability to pass a scope as a technical response. The reported technical response rate ranged from $79 \%$ to $100 \%$, whereas that using scope passage as the definition, which is the most obvious outcome, was $56 \%$ to $100 \%$. Clinical efficacy was also determined based on the definition used in each study. Most investigators defined clinical efficacy as disappearance of stenotic symptoms, such as abdominal pain, nausea and abdominal boating. Recently, a visual analogue scale (VAS) has been used as a more strict evaluation method for confirmation of symptom relief. ${ }^{30}$ In published studies, clinical efficacy was achieved in $42 \%$ to $90 \%$ of patients.

The safety profile of EBD is important as a minimally invasive treatment. Perforation is one of the major concerns during this procedure, because it usually requires emergent surgery. No perforations were reported in four studies, ${ }^{13,19,21,23}$ although the remaining 13 studies reported perforations. $^{8-12,14-18,20,22,24}$ The rate of perforation was $<10 \%$ in all but two studies, ${ }^{9,14}$ in which they were observed in $10 \%$ and $11 \%$ of subjects, respectively. EBD is thus recognized as a safe endoscopic treatment in patients with $\mathrm{CD}$, since the shortterm technical and clinical efficacy rates are relatively high and complication rates are low. Therefore, EBD for colorectal and ileo-colonic anastomotic Crohn's strictures may be as good a therapeutic option as surgical intervention, at least for the temporary relief of stenotic symptoms.

\section{Long-Term Outcomes}

Clinicians who treat CD patients with intestinal strictures should also be concerned about the long-term outcomes of EBD. Although this important outcome is generally assessed on the basis of symptom recurrence and surgery-free survival, in this review, only symptom recurrence was adopted as a criterion to evaluate long-term efficacy. The relapse rate of obstructive symptoms after EBD has been reported to be $24 \%$ to $79 \%$. Approximately $60 \%$ of published studies reported that $50 \%$ or more of patients showed signs of relapse after initial EBD. In patients with recurrence of symptoms, redilation or surgical intervention should be performed. According to a meta-analysis that summarized 25 studies, including 1,089 patients and 2,664 dilations, the proportion of patients who required further dilation at 1,2, and 5 years of followup was $31.6 \%, 25.9 \%$, and $1.7 \%$, respectively. ${ }^{35}$ Overall, the cumulative proportion of patients requiring further dilation over 5 years was $80.6 \%$. Although EBD enables avoidance of surgery, the need for frequent redilation is a burden for both clinicians and patients. Hence, further information on patients who are likely to develop recurrence of symptoms is desirable. In fact, several studies have analyzed the predictors of redilation or surgery after EBD. Using stepwise logistic regression in a systematic review, Hassan et al. ${ }^{36}$ pointed out that stricture length of $4 \mathrm{~cm}$ or more was the only signifi- 


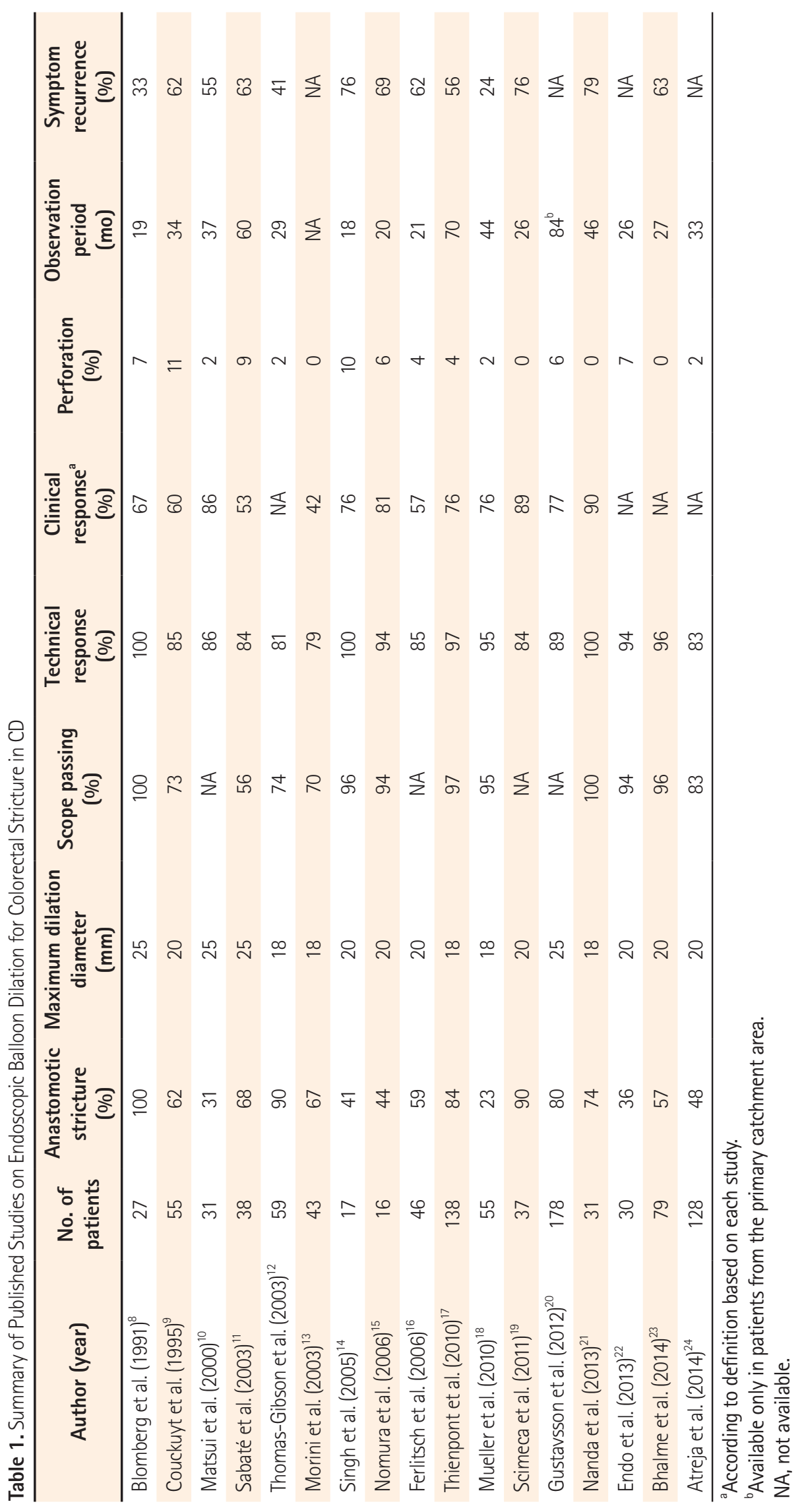


cant risk factor for recurrence (OR, 4.01; 95\% CI, 1.16-13.8; $P<0.028)$. On the other hand, other factors, such as the nature of strictures (naïve vs. postsurgical, active CD vs. inactive CD lesion), scope passage after EBD, and the method of graded dilation were not associated with treatment outcome. ${ }^{35,36}$ Smoking is thought to be a risk factor for surgery after EBD. ${ }^{37}$ Thus, careful follow-up after EBD is needed for patients with risk factors.

\section{Methods for More Effective and Safe EBD}

Several reports described methods for more effective and safe EBD. Steroid injection into the stricture site after EBD was reported as a useful method for enhancing efficacy. ${ }^{38,39}$ However, East et al. ${ }^{40}$ concluded that this method did not reduce the time to redilation after EBD of Crohn's ileo-colonic anastomotic strictures. Hence, further analysis including a large randomized controlled trial is essential to confirm the utility of steroid injection after EBD.

Carbon dioxide $\left(\mathrm{CO}_{2}\right)$ insufflation is thought to be useful for the safe and smooth performance of EBD. $\mathrm{CO}_{2}$ insufflation is associated with rapid absorption resulting in less gas retention, which results in less pain during and after endoscopic treatment. ${ }^{41-43}$ As EBD can be time-consuming, $\mathrm{CO}_{2}$ insufflation is more likely to have good short-term outcomes than air insufflation. ${ }^{44}$

\section{EBD USING BAE FOR SMALL BOWEL STRICTURES}

EBD using BAE for small bowel strictures is almost the same as EBD for colorectal and ileo-colonic strictures in terms of procedure and technique. However, special insertion techniques for deeper portions of the small intestine in BAE are necessary. Moreover, the proper insertion route should be determined according to the results of small bowel imaging. In addition, there are some technical difficulties, compared to EBD performed with a conventional colonoscope. For instance, it is not easy to stabilize the tip of the scope and to maintain a good visual field because of the limited space available, severe angulation, and adhesion in the small intestine. Therefore, EBD for small bowel strictures in $\mathrm{CD}$ is still challenging, although reports regarding this endoscopic intervention have recently increased, mainly from Japan (Table 2) ${ }^{27-34}$ According to published studies, maximum dilation diameter for strictures of the small intestine varied from $15 \mathrm{~mm}$ to $20 \mathrm{~mm}$ which is relatively small compared to EBD for colorectal and ileo-colonic strictures. It is speculated that the reasons for the small dilation diameter

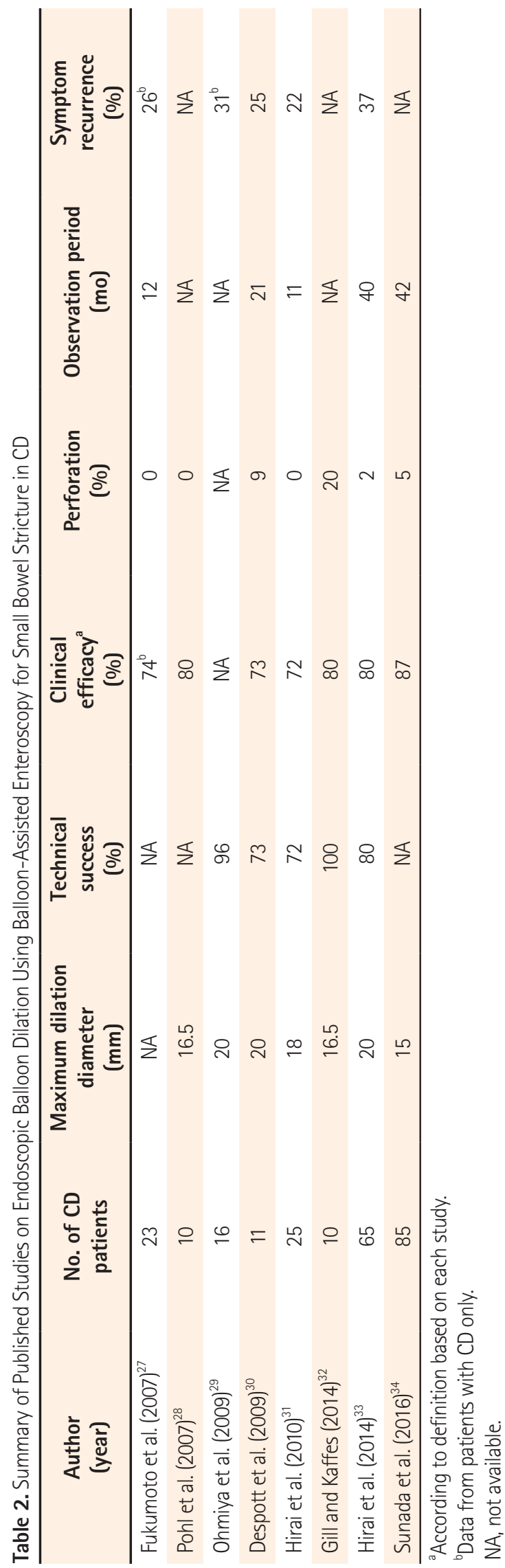


are more severe strictures and the operator's concerns regarding perforation.

There are also no standardized definitions of short-term success of EBD for small bowel strictures. Despite the cited difficulties, the reported technical success rate is $72 \%$ to $100 \%$, which is similar to the results of EBD for colorectal and ileo-colonic strictures. While clinical efficacy was evaluated based on the patient's obstructive symptoms in almost all studies, Despott et al. ${ }^{30}$ adopted the VAS score for abdominal pain in their evaluation. The clinical success rate was $>70 \%$ (72\%-87\%) overall, which is comparable to published data on EBD for colorectal strictures. Perforations occurred in $0 \%$ to $10 \%$ of subjects in most published studies, although one small cohort reported a perforation rate of $20 \%(2 / 10) .^{32}$ Judging from these results, EBD using BAE for small bowel strictures is equal to EBD using a conventional colonoscope in terms of short-term efficacy and safety.

There is insufficient reliable data on the long-term outcomes of the procedure for small bowel strictures, because the observation periods were very short, as shown in Table 2. However, in two studies that investigated the long-term outcomes of EBD for small bowel strictures, the average observation period after initial EBD was more than 3 years. ${ }^{33,34}$ Hirai et al. ${ }^{33}$ reported that the cumulative surgery-free rate in
65 subjects was $79 \%$ and $73 \%$ at 2 and 3 years, respectively. However, the cumulative redilation-free rate in their analysis was $64 \%$ and $47 \%$ at 2 and 3 years. This rate was similar to the results reported in previous studies evaluating EBD for colonic and ileo-colonic anastomosis. Therefore, the need for frequent redilation seems to be an issue with EBD regardless of the target stricture site. According to a recent study published by Sunada et al., ${ }^{34}$ the cumulative surgery-free rate after initial EBD for small bowel strictures in CD was 87.3\% at 1 year and $78.1 \%$ at 3 years. Even though the cumulative surgery-free rate was similar to that in the study by Hirai et al., ${ }^{33}$ they included patients with fistulas as subjects, and the presence of a fistula was significantly associated with the need for surgical intervention (hazard ratio, 5.50; 95\% CI, 2.16-14.0; $P=0.01)$. Hence, for small bowel strictures with fistula, EBD should be considered only in patients who are likely to benefit (e.g., for completion of important life events) from postponement of surgery.

The tendency for CD patients to have multiple small bowel strictures is one of the limitations of EBD using BAE. In fact, the proportion of patients with multiple small bowel strictures was high in published studies. Although the therapeutic strategy for this situation is not established, in my opinion, in the absence of exclusion criteria such as fistulas,
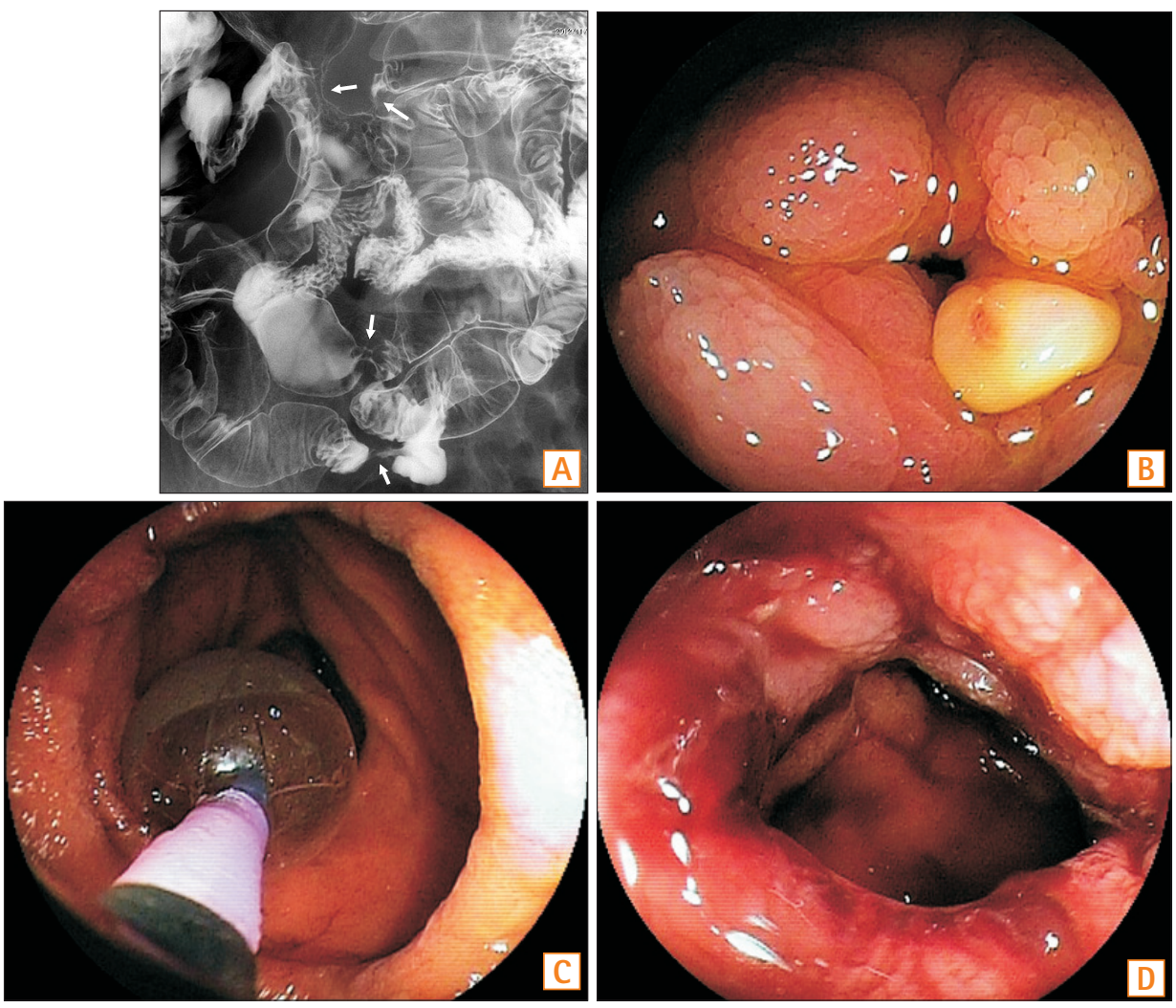

Fig. 1. (A) Double-contrast small bowel enterography showed multiple intestinal strictures (arrows) in the ileum. (B) Endoscopic findings of the most severe small bowel stricture. (C) Endoscopic findings during inflation of through-the-scope balloon catheter. Endoscopic balloon dilation (EBD) could be performed for all small bowel strictures. (D) Small bowel stricture markedly improved after EBD. Although two additional EBD sessions were needed, clinical efficacy (defined as no stenotic symptoms and no need for surgery) has been maintained for 5 years after the initial EBD. 
EBD can be attempted before performing surgery (Fig. 1). Further strict analysis will be needed for confirmation of efficacy of EBD for multiple small bowel strictures.

The disease behavior of CD has changed over time, with most patients having a stricturing type or a penetrating type. ${ }^{45,46}$ In particular, since patients with ileal type CD tend to have small bowel strictures, performing EBD using BAE would be more meaningful to improve their quality of life. However, as described above, there is only limited evidence regarding the utility of this endoscopic intervention in patients with small bowel strictures. Further studies confirming the efficacy and safety of this procedure are desirable. In fact, a nationwide, multicenter, open-label, prospective cohort study is now ongoing in Japan. According to the results of interim analysis in this study, the short-term success rate using VAS score as an indicator was $69 \%$, and no perforations were reported. ${ }^{47}$ The results also indicated equal efficacy and safety compared to published studies. The full data of this study will be published in the near future.

\section{ISSUES AND FUTURE DIRECTIONS}

Many issues should be addressed regarding EBD in CD. First, it is difficult to state whether EBD or surgery is best for release of intestinal strictures. Direct comparison between these two interventions is not easy, due to the differences in invasiveness and the goal of each treatment. Surgical interventions are superior to EBD in terms of certainty. According to a small retrospective cohort study, the proportion of patients who required any reintervention during follow-up was significantly lower in the surgical group than in the EBD group (OR, 5.62; 95\% CI, 1.66-19.01; $P=0.005) .{ }^{48}$ However, surgical interventions are inferior to EBD in terms of both the invasiveness and relapse at the anastomotic site. Strictureplasty has been performed for intestinal strictures in CD as a small bowel-saving strategy. ${ }^{49-52}$ Although the postoperative recurrence rate after strictureplasty is comparable to that of surgical resection, clinicians should also pay attention to the risk of malignancy in the preserved bowel. Since the performance of a randomized controlled study to compare EBD and surgical intervention is extremely difficult, gastroenterologists need to select an intervention method for intestinal strictures in CD by considering various factors (e.g., patients background, status of stricture, disease activity, length of small intestine, and so on) on a case by case basis.

The basic treatments for CD are not endoscopic or surgical interventions but medical therapies to control disease activity and improve quality of life. There are many effective medical treatments for CD, such as anti-tumor necrosis factor $\alpha$ antibody and immunomodulators. Additional candidates for treatments of CD will emerge in the future. Ideally, a combination of EBD and these effective medical treatments should be used for CD patients with intestinal strictures.

New endoscopic treatment modalities for Crohn's strictures such as the use of self-expanding metallic stents ${ }^{53,54}$ and needle-knife treatment ${ }^{55}$ have been reported. Implantation of metallic stents has mainly been used for patients with malignant gastrointestinal strictures because these enable improved food intake without surgery. As EBD has the limitation of loss of long-term efficacy, stents have been used for Crohn's strictures in several institutions. However, there are some disadvantages, such as inflammatory reactions to a foreign body, recurrent stricture due to mucosal growth into the stent, and perforation or hemorrhage with self-expansion. Therefore, further large-scale studies to confirm the efficacy and safety of stenting are needed. Needle-knife treatment is a limited option for Crohn's stricture. With only a few case reports, ${ }^{55,56}$ its efficacy is still uncertain and safety is questionable. Further experience is necessary in order to apply this intervention in CD patients with intestinal strictures.

\section{REFERENCES}

1. Harper PH, Fazio VW, Lavery IC, et al. The long-term outcome in Crohn's disease. Dis Colon Rectum 1987;30:174-179.

2. Munkholm P, Langholz E, Davidsen M, Binder V. Disease activity courses in a regional cohort of Crohn's disease patients. Scand J Gastroenterol 1995;30:699-706.

3. Rungoe C, Langholz E, Andersson M, et al. Changes in medical treatment and surgery rates in inflammatory bowel disease: a nationwide cohort study 1979-2011. Gut 2014;63:1607-1616.

4. Bernell O, Lapidus A, Hellers G. Risk factors for surgery and postoperative recurrence in Crohn's disease. Ann Surg 2000;231:38-45.

5. Hellers G. Crohn's disease in Stockholm county 1955-1974: a study of epidemiology, results of surgical treatment and longterm prognosis. Acta Chir Scand Suppl 1979;490:1-84.

6. Poggioli G, Pierangeli F, Laureti S, Ugolini F. Review article: indication and type of surgery in Crohn's disease. Aliment Pharmacol Ther 2002;16 Suppl 4:59-64.

7. Matsui T, Hatakeyama S, Ikeda K, Yao T, Takenaka K, Sakurai T. Long-term outcome of endoscopic balloon dilation in obstructive gastroduodenal Crohn's disease. Endoscopy 1997;29:640-645.

8. Blomberg B, Rolny P, Järnerot G. Endoscopic treatment of anastomotic strictures in Crohn's disease. Endoscopy 1991;23:195-198. 
9. Couckuyt H, Gevers AM, Coremans G, Hiele M, Rutgeerts P. Efficacy and safety of hydrostatic balloon dilatation of ileocolonic Crohn's strictures: a prospective longterm analysis. Gut 1995;36:577-580.

10. Matsui T, Ikeda K, Tsuda S, et al. Long-term outcome of endoscopic balloon dilation in obstructive gastrointestinal Crohn's disease: a prospective long-term study. Diagn Ther Endosc 2000;6:67-75.

11. Sabaté JM, Villarejo J, Bouhnik Y, et al. Hydrostatic balloon dilatation of Crohn's strictures. Aliment Pharmacol Ther 2003;18:409-413.

12. Thomas-Gibson S, Brooker JC, Hayward CM, Shah SG, Williams CB, Saunders BP. Colonoscopic balloon dilation of Crohn's strictures: a review of long-term outcomes. Eur J Gastroenterol Hepatol 2003;15:485-488.

13. Morini S, Hassan C, Lorenzetti R, et al. Long-term outcome of endoscopic pneumatic dilatation in Crohn's disease. Dig Liver Dis 2003;35:893-897.

14. Singh VV, Draganov P, Valentine J. Efficacy and safety of endoscopic balloon dilation of symptomatic upper and lower gastrointestinal Crohn's disease strictures. J Clin Gastroenterol 2005;39:284-290.

15. Nomura E, Takagi S, Kikuchi T, et al. Efficacy and safety of endoscopic balloon dilation for Crohn's strictures. Dis Colon Rectum 2006;49(10 Suppl):S59-S67.

16. Ferlitsch A, Reinisch W, Püspök A, et al. Safety and efficacy of endoscopic balloon dilation for treatment of Crohn's disease strictures. Endoscopy 2006;38:483-487.

17. Thienpont C, D'Hoore A, Vermeire S, et al. Long-term outcome of endoscopic dilatation in patients with Crohn's disease is not affected by disease activity or medical therapy. Gut 2010;59:320-324.

18. Mueller T, Rieder B, Bechtner G, Pfeiffer A. The response of Crohn's strictures to endoscopic balloon dilation. Aliment Pharmacol Ther 2010;31:634-639.

19. Scimeca D, Mocciaro F, Cottone M, et al. Efficacy and safety of endoscopic balloon dilation of symptomatic intestinal Crohn's disease strictures. Dig Liver Dis 2011;43:121-125.

20. Gustavsson A, Magnuson A, Blomberg B, Andersson M, Halfvarson J, Tysk C. Endoscopic dilation is an efficacious and safe treatment of intestinal strictures in Crohn's disease. Aliment Pharmacol Ther 2012;36:151-158.

21. Nanda K, Courtney W, Keegan D, et al. Prolonged avoidance of repeat surgery with endoscopic balloon dilatation of anastomotic strictures in Crohn's disease. J Crohns Colitis 2013;7:474-480.

22. Endo K, Takahashi S, Shiga H, Kakuta Y, Kinouchi Y, Shimosegawa T. Short and long-term outcomes of endoscopic balloon dilatation for Crohn's disease strictures. World J Gastroenterol 2013;19:86-91.
23. Bhalme M, Sarkar S, Lal S, Bodger K, Baker R, Willert RP. Endoscopic balloon dilatation of Crohn's disease strictures: results from a large United Kingdom series. Inflamm Bowel Dis 2014;20:265-270.

24. Atreja A, Aggarwal A, Dwivedi S, et al. Safety and efficacy of endoscopic dilation for primary and anastomotic Crohn's disease strictures. J Crohns Colitis 2014;8:392-400.

25. Yamamoto H, Kita H, Sunada K, et al. Clinical outcomes of double-balloon endoscopy for the diagnosis and treatment of small-intestinal diseases. Clin Gastroenterol Hepatol 2004;2:1010-1016.

26. Yamamoto H, Yano T, Ohmiya N, et al. Double-balloon endoscopy is safe and effective for the diagnosis and treatment of small-bowel disorders: prospective multicenter study carried out by expert and non-expert endoscopists in Japan. Dig Endosc 2015;27:331-337.

27. Fukumoto A, Tanaka S, Yamamoto H, et al. Diagnosis and treatment of small-bowel stricture by double balloon endoscopy. Gastrointest Endosc 2007;66(3 Suppl):S108-S112.

28. Pohl J, May A, Nachbar L, Ell C. Diagnostic and therapeutic yield of push-and-pull enteroscopy for symptomatic small bowel Crohn's disease strictures. Eur J Gastroenterol Hepatol 2007;19:529-534.

29. Ohmiya N, Arakawa D, Nakamura M, et al. Small-bowel obstruction: diagnostic comparison between double-balloon endoscopy and fluoroscopic enteroclysis, and the outcome of enteroscopic treatment. Gastrointest Endosc 2009;69:84-93.

30. Despott EJ, Gupta A, Burling D, et al. Effective dilation of smallbowel strictures by double-balloon enteroscopy in patients with symptomatic Crohn's disease (with video). Gastrointest Endosc 2009;70:1030-1036.

31. Hirai F, Beppu T, Sou S, Seki T, Yao K, Matsui T. Endoscopic balloon dilatation using double-balloon endoscopy is a useful and safe treatment for small intestinal strictures in Crohn's disease. Dig Endosc 2010;22:200-204.

32. Gill RS, Kaffes AJ. Small bowel stricture characterization and outcomes of dilatation by double-balloon enteroscopy: a singlecentre experience. Therap Adv Gastroenterol 2014;7:108-114.

33. Hirai F, Beppu T, Takatsu N, et al. Long-term outcome of endoscopic balloon dilation for small bowel strictures in patients with Crohn's disease. Dig Endosc 2014;26:545-551.

34. Sunada K, Shinozaki S, Nagayama M, et al. Long-term outcomes in patients with small intestinal strictures secondary to Crohn's disease after double-balloon endoscopy-assisted balloon dilation. Inflamm Bowel Dis 2016;22:380-386.

35. Morar PS, Faiz O, Warusavitarne J, et al. Systematic review with meta-analysis: endoscopic balloon dilatation for Crohn's disease strictures. Aliment Pharmacol Ther 2015;42:1137-1148. 
36. Hassan C, Zullo A, De Francesco V, et al. Systematic review: endoscopic dilatation in Crohn's disease. Aliment Pharmacol Ther 2007;26:1457-1464.

37. Gustavsson A, Magnuson A, Blomberg B, Andersson M, Halfvarson J, Tysk C. Smoking is a risk factor for recurrence of intestinal stricture after endoscopic dilation in Crohn's disease. Aliment Pharmacol Ther 2013;37:430-437.

38. Brooker JC, Beckett CG, Saunders BP, Benson MJ. Long-acting steroid injection after endoscopic dilation of anastomotic Crohn's strictures may improve the outcome: a retrospective case series. Endoscopy 2003;35:333-337.

39. Di Nardo G, Oliva S, Passariello M, et al. Intralesional steroid injection after endoscopic balloon dilation in pediatric Crohn's disease with stricture: a prospective, randomized, double-blind, controlled trial. Gastrointest Endosc 2010;72:1201-1208.

40. East JE, Brooker JC, Rutter MD, Saunders BP. A pilot study of intrastricture steroid versus placebo injection after balloon dilatation of Crohn's strictures. Clin Gastroenterol Hepatol 2007;5:1065-1069.

41. Hirai F, Beppu T, Nishimura T, et al. Carbon dioxide insufflation compared with air insufflation in double-balloon enteroscopy: a prospective, randomized, double-blind trial. Gastrointest Endosc 2011;73:743-749.

42. Wang WL, Wu ZH, Sun Q, et al. Meta-analysis: the use of carbon dioxide insufflation vs. room air insufflation for gastrointestinal endoscopy. Aliment Pharmacol Ther 2012;35:1145-1154.

43. Lenz P, Meister T, Manno M, et al. CO2 insufflation during single-balloon enteroscopy: a multicenter randomized controlled trial. Endoscopy 2014;46:53-58.

44. Hirai F, Matsui T, Yao K, Sou S, Seki T. Efficacy of carbon dioxide insufflation in endoscopic balloon dilation therapy by using double balloon endoscopy. Gastrointest Endosc 2007;66(3 Suppl):S26-S29.

45. Cosnes J, Cattan S, Blain A, et al. Long-term evolution of disease behavior of Crohn's disease. Inflamm Bowel Dis 2002;8:244250.
46. Sato Y, Matsui T, Yano Y, et al. Long-term course of Crohn's disease in Japan: incidence of complications, cumulative rate of initial surgery, and risk factors at diagnosis for initial surgery. J Gastroenterol Hepatol 2015;30:1713-1719.

47. Hirai F. Efficacy of endoscopic balloon dilation for small bowel strictures in patients with Crohn's disease. Proceedings of the 4th Annual Meeting of Asian Organization for Crohn's \& Colitis; 2016 July 7-9; Kyoto, Japan.

48. Greener T, Shapiro R, Klang E, et al. Clinical outcomes of surgery versus endoscopic balloon dilation for stricturing Crohn's disease. Dis Colon Rectum 2015;58:1151-1157.

49. Yamamoto T, Keighley MR. Long-term results of strictureplasty for ileocolonic anastomotic recurrence in Crohn's disease. J Gastrointest Surg 1999;3:555-560.

50. Sampietro GM, Cristaldi M, Maconi G, et al. A prospective, longitudinal study of nonconventional strictureplasty in Crohn's disease. J Am Coll Surg 2004;199:8-20.

51. Futami K, Arima S. Role of strictureplasty in surgical treatment of Crohn's disease. J Gastroenterol 2005;40 Suppl 16:35-39.

52. Yamamoto T, Fazio VW, Tekkis PP. Safety and efficacy of strictureplasty for Crohn's disease: a systematic review and metaanalysis. Dis Colon Rectum 2007;50:1968-1986.

53. Attar A, Maunoury V, Vahedi K, et al. Safety and efficacy of extractible self-expandable metal stents in the treatment of Crohn's disease intestinal strictures: a prospective pilot study. Inflamm Bowel Dis 2012;18:1849-1854.

54. Loras C, Pérez-Roldan F, Gornals JB, et al. Endoscopic treatment with self-expanding metal stents for Crohn's disease strictures. Aliment Pharmacol Ther 2012;36:833-839.

55. Kerkhof M, Dewint P, Koch AD, van der Woude CJ. Endoscopic needle-knife treatment of refractory ileo-ascending anastomotic stricture. Endoscopy 2013;45 Suppl 2:E57-E58.

56. Shen B, Lian L, Kiran RP, et al. Efficacy and safety of endoscopic treatment of ileal pouch strictures. Inflamm Bowel Dis 2011;17:2527-2535. 